\title{
Cơ sở lý thuyết và thực tiễn đo lường hiệu quả môi trường trong sản xuất nông nghiệp: Trường hợp nuôi tôm vùng chuyển đổi tại Kiên Giang
}

\section{Theoretical and empirical frameworks for measuring environmental efficiency in agricultural production: A case study of shrimp farming in transforming areas of Kien Giang province}

\author{
Nguyễn Thùy Trang ${ }^{1 *}$, Huỳnh Việt Khải ${ }^{1}$, Võ Hồng Tú ${ }^{1}$, Trần Minh Hải ${ }^{2}$ \\ ${ }^{1}$ Trường Đại học Cần Thơ, Việt Nam \\ ${ }^{2}$ Trường Đại học An giang, Việt Nam \\ *Tác giả liên hệ, Email: nttrang@ctu.edu.vn
}

THÔNG TIN

DOI:10.46223/HCMCOUJS. econ.vi.14.1.507.2019

Ngày nhận: 14/10/2018

Ngày nhận lại: 04/12/2018

Duyệt đăng: 14/01/2019

\section{Tù khóa:}

hiệu quả kỹ thuật, hiệu quả môi trường, những đầu vào có ảnh hưởng xấu đến môi trường, nuôi tôm

\section{TÓM TẮT}

Hiệu quả môi trường là một trong những khía cạnh quan trọng quyết định đến tính bền vững của các mô hình sản xuất nông nghiệp. Do vậy, việc đo lường chính xác chỉ số này bằng mô hình kinh tế lượng có vai trò quan trọng trong đề xuất chính sách. Bài viết giới thiệu đo lường hiệu quả môi trường bằng cách tiếp cận phân tích giới hạn biên ngẫu nhiên (Stochastic frontier analysis). Hiệu quả môi trường bằng cách tiếp cận sản xuất biên ngẫu nhiên phản ánh khả năng giảm các đầu vào có ảnh hưởng xấu đến môi trường trong điều kiện đầu vào thông thường và đầu ra cố định. Bằng cách sử dụng bộ số liệu của 67 nông hộ nuôi tôm thâm canh vùng chuyển đổi tỉnh Kiên Giang, kết quả nghiên cứu cho thấy hiệu quả môi trường trung bình của nông hộ là $52,79 \%$, cho thấy nông hộ vẫn chưa sử dụng hiệu quả các yếu tố đầu vào có ảnh hưởng xấu đến môi trường hay nói cách khác nông hộ có thể giảm khoảng $47,21 \%$ tổng lượng đầu vào các yếu tố thức ăn, thuốc và nhiên liệu. Kết quả nghiên cứu cũng cho thấy có sự khác biệt lớn về hiệu quả môi trường giữa các nông hộ nuôi tôm trên địa bàn nghiên cứu.

\begin{abstract}
Environmental efficiency is one of the key aspects that determine the sustainability of agricultural production models. Thus, accurate measurement of this indicator by econometric model plays an important role in policy recommendations. The paper introduces an approach to
\end{abstract}


Keywords:

technical efficiency, environmental efficiency, environmentally detrimental inputs, shrimp farming

measure environmental efficiency by using the stochastic frontier analysis (SFA). Environmental efficiency measured by SFA method reflects the possibility of reducing environmentally detrimental inputs while keeping normal inputs and output constant. By using the data set of 67 intensive shrimp farmers in Kien Giang province, the results show that the average environmental efficiency was $52.79 \%$, indicating that farmers have not used the environmentally detrimental inputs efficiently, or in other words the farmers could reduce about $47.21 \%$ of the total inputs feed, medicine and fuel. The results also show that there was a significant difference in environmental performance among shrimp farmers in the study sites.

\section{Giới thiệu}

Việt Nam là quốc gia trọng điểm về sản xuất nông nghiệp và đóng góp to lớn trong đảm bảo an ninh lương thực quốc gia cũng như thế giới, cụ thể hơn 6,5 triệu tấn gạo xuất khẩu mỗi năm (GSO, 2013). Tuy nhiên, theo nhiều đánh giá cho thấy đời sống của người dân sản xuất nông nghiệp vẫn còn gặp nhiều khó khăn, đặc biệt là vùng ven biển (B. T. Nguyen, Lam, \& Thach, 2012; C. D. Nguyen, 2011). Tình hình biến đổi khí hậu ngày càng nghiêm trọng (CarewReid, 2008; Wassmann, Nguyen, Chu, \& To, 2004) và sự bất ổn định về thị trường, giá bán thấp trong khi giá vật tư tăng cao làm cho việc thay đổi mổ hình sản xuất ở các nước đang phát triển diê̂n ra như là một hiện tượng tất yếu. Đồng bằng sông Cửu Long (ĐBSCL) là một trong ba đồng bằng chịu ảnh hưởng nặng nề nhất bởi biến đổi khí hậu. Trong thời gian gần đây nhiều nông dân canh tác mô hình lúa - tôm ở khu vực ĐBSCL, đặc biệt là tỉnh Kiên Giang đã chuyển đổi sang mô hình nuôi tôm nhằm kỳ vọng lợi nhuận cao hơn. Tuy nhiên, quá trình chuyển đổi yêu cầu đầu tư cao và sự chuẩn bị tốt về mặt kỹ thuật sản xuất, do vậy rủi ro xảy ra trong quá trình chuyển đổi là rất cao $(\mathrm{MARD}, 2014)$. Những khía cạnh chính của sự rủi ro này không chỉ đơn thuần về mặt kinh tế mà còn liên quan đến vấn đề ô nhiễm môi trường do người dân chưa có nhiều kỹ thuật trong quản lý, sử dụng kết hợp các yếu tố đầu vào có ảnh hưởng xấu đến môi trường (environmentally detrimental inputs) như thức ăn, thuốc, nhiên liệu, ....

Hiệu quả môi trường là một trong những khía cạnh quan trọng quyết định đến tính bền vững của các mô hình sản xuất nông nghiệp. Nền nông nghiệp của Việt Nam nói chung và ĐBSCL nói riêng đã trải qua một thời gian dài phát triển và có thể nói đã đạt đến giới hạn trên hay đơn giản hơn là việc gia tăng đầu vào sẽ không còn hiệu quả trong gia tăng năng suất sản xuất. Tuy nhiên, nhiều nông dân vẫn còn sản xuất theo kinh nghiệm và thiếu kiến thức về quản lý nên thường dẫn đến tình trạng kém hiệu quả hay sử dụng quá mức khuyến cáo các đầu vào có ảnh hưởng đến môi trường như phân, thuốc, nhiên liệu, ...Theo nhiều nghiên cứu cho thấy, vấn đề lạm dụng nông dược đã gây ảnh hưởng lớn đến môi trường và sức khỏe người sản xuất cũng như người tiêu dùng (Heong \& Hardy, 2009; D. H. Nguyen \& Tran, 1999).

Hiệu quả môi trường là một thuật ngữ khá mới, được đề xuất sử dụng trong thời gian gần đây để đo lường thực trạng sử dụng các đầu vào có ảnh hưởng xấu đến môi trường như nhiên liệu, phân hóa học, thuốc bảo vệ thực vật, ... Thuật ngữ này đầu tiên được đề xuất bởi 
Reinhard, Lovell, và Thijssen (1999) nhằm đo lường hiệu quả môi trường cho mô hình nuôi bò sữa. Do nhu cầu về các sản phẩm sạch và an toàn ngày càng tăng cũng như tình hình ô nhiễm môi trường sẽ trở nên nghiêm trọng nếu như lạm dụng nông dược diễn ra trong một thời gian dài, nhu cầu đo lường hiệu quả môi trường của các hoạt động sản xuất nông nghiệp là rất cần thiết.

Theo kết quả lược khảo tài liệu cho thấy, hiện ở Việt Nam nói chung và khu vực ĐBSCL nói riêng vẫn chưa có nhiều nghiên cứu về đo lường hiệu quả môi trường, đặc biệt là cho lĩnh vực thủy sản. Một số nghiên cứu điển hình về đo lường hiệu quả môi trường chủ yếu tập trung cho lĩnh vực sản xuất lúa và trồng trà $(H$. B. Nguyen, Takahashi, \& Yabe, 2016; Vo, 2017; Vo \& Yabe, 2015; Vo, Nguyen, Takahashi, Kopp, \& Yabe, 2018).

Dựa trên kết quả lược khảo, có hai cách tiếp cận chính được sử dụng để đo lường hiệu quả môi trường là phương pháp phân tích vỏ bọc dữ liệu (DEA) và phân tích giới hạn sản xuất biên ngẫu nhiên (SFA). Do DEA là một cách tiếp cận dựa trên mô hình tuyến tính (mathematic programming) và phi tham số (non-parametric) nên kết quả ước lượng hiệu quả không thể tách các tác động nhiễu trong khi đó cách tiếp cận SFA dựa trên mô hình kinh tế lượng nên có thể khắc phục được nhược điểm này của DEA (Vo \& Yabe, 2015).

Bên cạnh hai cách tiếp cận DEA và SFA, một số phương pháp đo lường hiệu quả môi trường hay tác động môi trường giản đơn khác cũng thường được các nhà quản lý áp dụng như:

- Đối với lĩnh vực môi trường: dựa vào tổng lượng phát thải và lượng phát thải chưa được xử lý ra môi trường, ... (Luật bảo vệ môi trường, 2014).

- Đối với lĩnh vực sản xuất nông nghiệp: so sánh đơn thuần tổng lượng phân, thuốc hoặc tổng lượng nước/hecta giữa các hộ/nhóm hộ, ... (Dang, Nguyen, \& Nguyen, 2007; H. B. Nguyen \& Yabe, 2017).

Tuy nhiên những phương pháp đo lường giản đơn này chưa xem xét đến mối quan hệ giữa đầu vào và đầu ra của quá trình sản xuất cũng như khả năng thay thế kỹ thuật biên giữa các đầu vào nên kết quả đánh giá thiếu tính chính xác (Billi, Canitano, \& Quarto, 2007; Sharma, Molden, \& Cook, 2015). Từ những lý do này, để góp phần giới thiệu phương pháp đo lường mới có độ tin cậy cao hơn, bài viết tập trung trình bày phương pháp đo lường hiệu quả môi trường bằng cách tiếp cận SFA.

Xuất phát từ thực trạng trên, bài viết tập trung vào hai nội dung chính: thứ nhất là giới thiệu cơ sở lý thuyết cũng như khung phân tích đo lường hiệu quả môi trường để giúp người đọc có thể hệ thống hóa được phương pháp cũng như tiến trình thực hiện đo lường. Thứ hai là ứng dụng phương pháp vào đo lường hiệu quả môi trường cho trường hợp nuôi trồng thủy sản, cụ thể là mô hình nuôi tôm thâm canh trên nền đất chuyển đổi tại tỉnh Kiên Giang để giúp người đọc có thể dễ dàng hệ thống hóa và ứng dụng cho các trường hợp nghiên cứu khác về đo lường hiệu quả môi trường.

\section{Phương pháp nghiên cứu}

\subsection{Cơ sở lý thuyết đo luờng hiệu quả môi truò̀ng}

Pittman (1983) được xem là người đầu tiên quan tâm về vấn đề môi trường khi ước lượng hiệu quả của hoạt động sản xuất thông qua nghiên cứu về "So sánh năng suất sản xuất đa khía cạnh cùng với nhũ̃ng đầu ra không mong đọi (Multilateral Productivity Comparisons with Undesirable Outputs)". Trong nghiên cứu này tác giả xem xét khía cạnh môi trường là một 
đầu ra không mong đợi của hoạt động sản xuất. Tác giả đã phát triển thêm từ thuật ngữ “Chỉ số sản xuất đa khí cạnh translog (translog multilateral productivity index)" của Caves, Christensen, và Diewert (1982). Chỉ số hiệu quả này xem xét vấn đề ô nhiễm thông qua hai đầu ra không mong đợi là ô nhiễm nước và ô nhiễm không khí từ hoạt động sản xuất. Nghiên cứu định nghĩa chỉ số hiệu quả về môi trường là khả năng tăng/giảm của đầu ra mong đợi và giảm/tăng của đầu ra không mong đợi. Kết quả từ nghiên cứu đã đóng vai trò quan trọng cho hoạch định chính sách trong bối cảnh đánh đổi giữa đầu ra mong đợi và đầu ra không mong đợi. Tuy nhiên, đo lường đầu ra không mong đợi là một công việc khó khăn, đặc biệt trong hoạt động sản xuất nông nghiệp.

Färe, Grosskopf, Lovell, và Pasurka (1989) đã đề xuất một thuật ngữ tạm dịch là "chỉ số hiệu quả sản xuất hy-péc-pôn cải tiến (enhanced hyperbolic productive efficiency measure)". Thuật ngữ này xem xét đồng thời sự khác biệt về khả năng tăng đầu ra mong đợi tối đa, khả năng giảm đầu ra không mong đợi tối đa và cùng lúc giảm các yếu tố đầu vào. Tuy nhiên, nghiên cứu này đề xuất phương pháp đo lường bằng DEA nên không thể tách các tác động nhiễu ra khỏi việc đo lường hiệu quả sản xuất. Thêm vào đó, đo lường đầu ra không mong đợi là một công việc khó khăn cho hoạt động sản xuất nông nghiệp.

Từ những hạn chế trên, Reinhard và cộng sự (1999) đã xem xét vấn đề môi trường ở khía cạnh đầu vào của hoạt động sản xuất gồm (e.g., phân đạm, phân lân và nhiên liệu) đề từ đó đo lường hiệu quả môi trường. Do các đầu vào có ảnh hưởng đến môi trường như phân bón hóa học, thuốc trừ sâu, nhiên liệu, ... có mối quan hệ mật thiết với đầu ra không mong đợi (ô nhiễm), nên tối thiểu hóa đầu ra không mong đợi có thể được thực hiện thông qua tối thiểu hóa các đầu vào có ảnh hưởng đến môi trường. Như vậy, thông qua chỉ số hiệu quả môi trường ta có thể thấy được mức độ lạm dụng hay khả năng giảm các đầu vào có ảnh hưởng xấu đến môi trường, trong điều kiện đầu ra và các đầu vào khác cố định. Như vậy để tính được hiệu quả môi trường thì trước tiên ta phải tính được hiệu quả kỹ thuật định hướng đầu ra. Hiệu quả môi trường về mặt phương pháp ước lượng gần giống với hiệu quả kỹ thuật định hướng đầu vào nhưng khác ở điểm là hiệu quả môi trường chỉ xem xét mức không hiệu quả của các yếu gây ảnh hưởng xấu đến môi trường. Hiệu quả môi trường được mô tả cụ thể bằng Hình 1 và 2.

Giả sử có hai yếu tố đầu vào $X$ (đầu vào thông thường) và $Z$ (đầu vào có ảnh hưởng đến môi trường). Như vậy, hàm sản xuất giới hạn ngẫu nhiên có thể được ước lượng và thể hiện thông qua $\mathrm{OX}_{\mathrm{R}} \mathrm{R}^{\mathrm{F}} \mathrm{Z}_{\mathrm{R}}$. Nông hộ $\mathrm{R}$ là điểm quan sát thực tế, sản xuất đầu ra ở $Y_{R}$ bằng cách sử dụng đầu vào $X_{R}$ và $Z_{R}$. ABCR là mặt phẳng đẳng lượng hay nói cách khác là với mọi cách kết hợp đầu vào sẽ cho ra cùng một mức sản lượng và nông hộ $R$ cũng thuộc mặt phẳng này. Theo kết quả Hình 2, chúng ta cũng có thể dễ dàng đo lường hiệu quả kỹ thuật định hướng đầu ra và hiệu quả kỹ thuật định hướng đầu vào, lần lượt với tỷ lệ $\left|O Y_{R}\right| /\left|O Y_{F}\right|$ và $\left|\mathrm{Y}_{\mathrm{R}} \mathrm{B}\right| /\left|\mathrm{Y}_{\mathrm{R}} \mathrm{R}\right|$. 


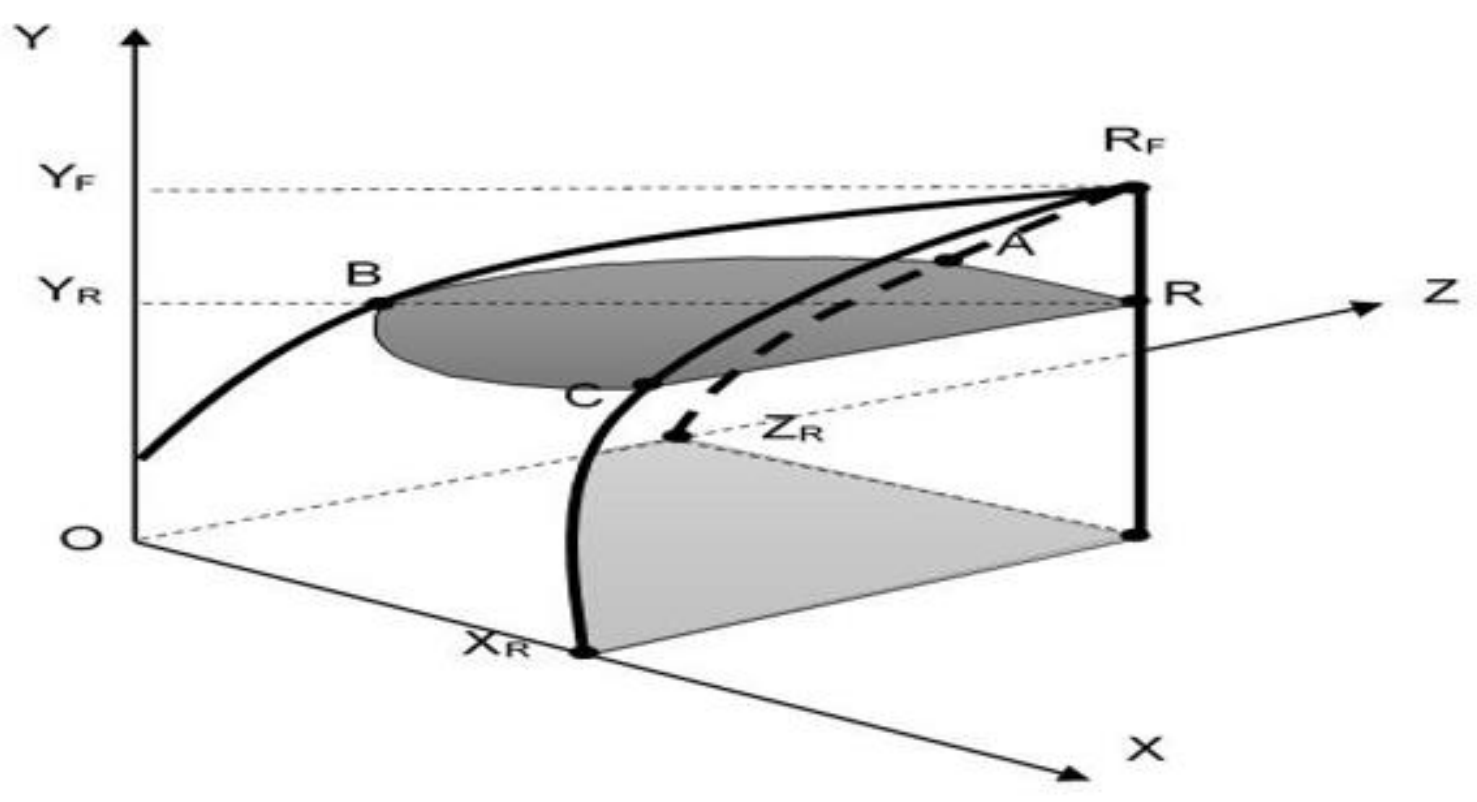

Hình 1. Mô phỏng đo lường hiệu quả môi trường bằng không gian ba chiều Nguồn: Vo, Yabe, Nguyen, và Huynh (2015)

Từ mặt phẳng $\mathrm{ABCR}$ này, để dễ hiểu chúng ta có thể chuyển sang không gian hai chiều như Hình 2.

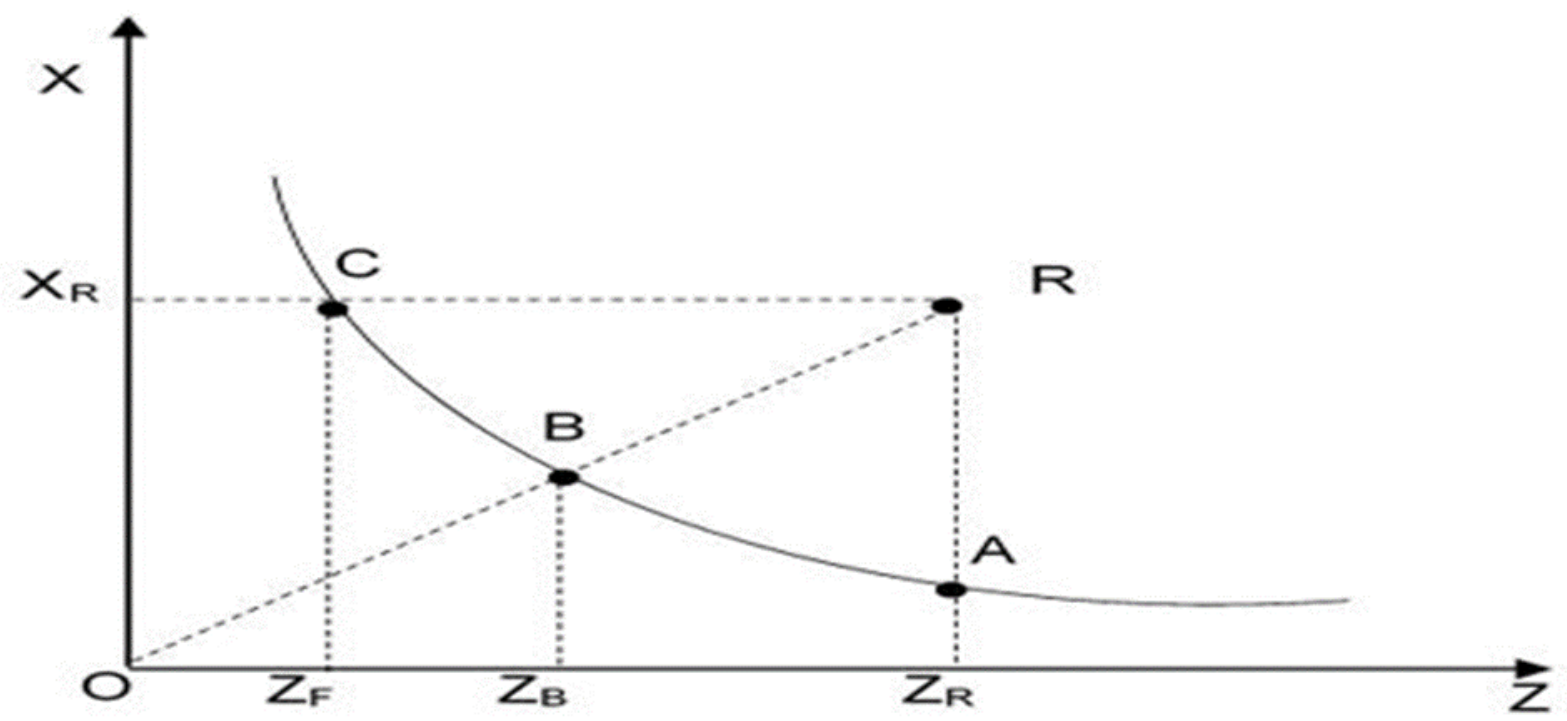

Hình 2. Mô phỏng mặt cắt đẳng lượng $\mathrm{ABCR}$

Nguồn: Vo và cộng sự (2015)

Từ Hình 2, ta có thể dễ dàng nhận thấy hiệu quả môi trường sẽ được phản ánh thông qua tỷ lệ $\left|O Z_{F}\right| /\left|O Z_{R}\right|$ do nông hộ $\mathrm{R}$ và nông hộ $\mathrm{C}$ có cùng lượng đầu ra nhưng mức độ sử dụng các yếu tố ảnh hưởng xấu đến môi trường $(Z)$ là khác nhau nên nông hộ $\mathrm{R}$ có thể giảm một lượng $Z_{F} Z_{R}$. 


\subsection{Khung phân tích đo lường hiệu quả môi trường}

Giả sử nông hộ sử dụng 02 nhóm đầu vào $(X$ và $Z)$ để sản xuất một đầu ra $Y\left(Y \in R_{+}\right)$, trong đó $X\left(X \in R_{+}\right)$là các đầu vào thông thường (conventional inputs) như vốn, lao động, giống,... và $Z\left(Z \in R_{+}\right)$là những đầu vào ảnh hưởng xấu đến môi trường như thức ăn, thuốc kháng sinh và nhiên liệu, thuốc bảo vệ thực vật và phân bón hóa học. Tùy vào các hoạt động sản xuất khác nhau mà nghiên cứu ứng dụng có thể tập trung vào những đầu vào ảnh hưởng đến môi trường khác nhau. Như vậy, hàm giới hạn sản xuất ngẫu nhiên được viết bằng phương trình tổng quát như sau:

$$
Y_{i}=f\left(X_{i}, Z_{i}, \beta, \alpha, \delta\right) \exp \left(v_{i}-u_{i}\right)
$$

Trong nghiên cứu hiệu quả môi trường sẽ sử dụng công thức hàm sản xuất giới hạn ngẫu nhiên (1) được viết lại dưới dạng translog theo phương trình (2) sau:

$$
\begin{aligned}
\operatorname{Ln} Y_{i}=\beta_{0}+\sum_{k} \beta_{k} \ln X_{k}+\sum_{m} \alpha_{m} \ln Z_{m} \\
+\frac{1}{2} \sum_{k} \sum_{n} \beta_{k n} \ln X_{k} \ln X_{n}+\frac{1}{2} \sum_{m} \sum_{h} \alpha_{m h} \ln Z_{m} \ln Z_{h} \\
+\sum_{k} \sum_{m}^{n} \delta_{k m} \ln X_{k} \ln Z_{m}+v_{i}-u_{i}
\end{aligned}
$$

Để có thể đo lường hiệu quả môi trường, Reinhard và cộng sự (1999); Reinhard, Lovell, và Thijssen (2000) cho $u_{i}$ bằng 0 với lý do là sau khi một nông hộ cắt giảm tất cả các đầu vào xấu cũng sẽ đạt được hiệu quả kỹ thuật. Sự cắt giảm các đầu vào xấu được thể hiện thông qua việc thay thế tất cả các đầu vào xấu $Z_{i m}$ trong phương trình (8) bằng $\Phi Z_{i m}$, trong đó $\Phi$ là hiệu quả môi trường hay $E E_{i}=\Phi_{i}$. Như vậy, sau khi cho $u_{i}=0$ và thế $Z_{i m}$ bằng $\Phi Z_{i m}$ trong phương trình (2), ta có:

$$
\begin{aligned}
\operatorname{Ln} Y_{i}=\beta_{0}+ & \sum_{k} \beta_{k} \ln X_{k}+\sum_{m} \alpha_{m} \ln \Phi Z_{m} \\
& +1 / 2 \sum_{k} \sum_{n} \beta_{k n} \ln X_{k} \ln X_{n}+1 / 2 \sum_{m} \sum_{h} \alpha_{m h} \ln \Phi Z_{m} \ln \Phi Z_{h} \\
& +\sum_{k} \sum_{m} \delta_{k m} \ln X_{k} \ln \Phi Z_{m}+v_{i}
\end{aligned}
$$

Do hiệu quả môi trường phản ánh khả năng giảm các đầu vào xấu trong điều kiện các đầu vào thông thường khác và đầu ra cố định. Điều này ngụ ý rằng phương trình (2) và phương trình (3) bằng nhau. Cho hai phương trình bằng nhau ta thu được phương trình (4) sau:

$$
\begin{aligned}
\sum_{m} \alpha_{m} \ln \Phi Z_{m} & -\sum_{m} \alpha_{m} \ln Z_{m} \\
& +1 / 2 \sum_{m} \sum_{h} \alpha_{m h} \ln \Phi Z_{m} \ln \Phi Z_{h}-1 / 2 \sum_{m} \sum_{h} \alpha_{m h} \ln Z_{m} \ln Z_{h} \\
& +\sum_{k} \sum_{m} \delta_{k m} \ln X_{k} \ln \Phi Z_{m}-\sum_{k} \sum_{m} \delta_{k m} \ln X_{k} \ln Z_{m}+u_{i}=0
\end{aligned}
$$


Lưu ý: $\ln \Phi_{\mathrm{i}}=\ln \Phi_{\mathrm{i}} \mathrm{Z}_{\mathrm{im}}-\ln \mathrm{Z}_{\mathrm{im}}=\ln \left(\frac{\Phi_{\mathrm{i}} \mathrm{Z}_{\mathrm{im}}}{\mathrm{Z}_{\mathrm{im}}}\right)=\ln E E_{i}$

Với vài phép biến đổi đơn giản của phương trình (4) ta thu được phương trình bậc 2 (5) sau

$$
a_{i}\left(\ln E E_{i}\right)^{2}+b_{i}\left(\ln E E_{i}\right)+u_{i}=0
$$

Trong đó $a_{i}=1 / 2 \sum_{m} \sum_{h} \alpha_{m h} \forall a_{i} \neq 0$;

$$
b_{i}=\sum_{m} \alpha_{m}+1 / 2 \sum_{m} \sum_{h} \alpha_{m h}\left(\ln Z_{m}+\ln Z_{h}\right)+\sum_{k} \sum_{m} \delta_{k m} \ln X_{k}
$$

Như vậy, nghiệm của phương trình bậc 2 (5) chính là hiệu quả môi trường. Nghiệm của phương trình (5) như sau:

$$
E E_{i}=\exp \left(\frac{-b_{i} \pm \sqrt{b_{i}^{2}-4 a_{i} u_{i}}}{2 a_{i}}\right)
$$

Phương trình (6) cho thấy có hai nghiệm tồn tại trong khi đó mỗi nông hộ chỉ có 01 chỉ số hiệu quả môi trường. Theo Reinhard và cộng sự (1999); Reinhard và cộng sự (2000); Reinhard và Thijssen (2000), do nông hộ đạt hiệu quả kỹ thuật $\left(u_{i}=0\right)$ thì phải đạt hiệu quả môi trường nên các tác giả chỉ lấy nghiệm $+\sqrt{ }$ của phương trình (6) trong đo lường hiệu quả môi trường. Như vậy, công thức tính hiệu quả môi trường cho từng nông hộ như sau:

$$
E E_{i}=\exp \left(\frac{-b_{i}+\sqrt{b_{i}^{2}-4 a_{i} u_{i}}}{2 a_{i}}\right)
$$

\subsection{Thu thập và phân tích số liệu}

Số liệu nghiên cứu được thu thập tại bốn huyện của tỉnh Kiên Giang gồm An Minh, An Biên, U Minh Thượng và Vĩnh Thuận. Đây là bốn huyện có diện tích chuyển đổi mô hình sản xuất nông nghiệp cao, cụ thể là từ mô hình lúa tôm sang tôm thâm canh. Đối tượng nghiên cứu là những nông hộ nuôi tôm thâm canh đã thực hiện chuyển đổi từ mô hình lúa - tôm. Để thực hiện thu thập số liệu, nghiên cứu đã tiến hành thảo luận với Phòng Nông nghiệp các huyện trong địa bàn nghiên cứu để lấy danh sách các nông dân đã chuyển đổi mô hình. Sau đó, nhóm nghiên cứu tiếp tục làm việc với các cán bộ nông nghiệp cấp xã để nhờ hỗ trợ dẫn đường phỏng vấn các nông hộ có tên trong danh sách. Tổng mẫu cho nghiên cứu là 67 hộ, đây cũng có thể được xem là tổng thể của nghiên cứu vì chỉ còn một số rất ít hộ không thể tiếp cận được do vắng nhà và lý do tâm lý không cho người lạ phỏng vấn.

Để thực hiện phân tích số liệu, nghiên cứu sử dụng phần mềm Stata ${ }^{\circledR} 12$.

\section{Kết quả và thảo luận}

\subsection{Mô hình úng dụng}

Dựa trên kết quả điều tra cũng như lược khảo tài liệu, nghiên cứu xác định các yếu tố đầu vào của quá trình nuôi tôm gồm 03 biến có ảnh hưởng đến môi trường là thức ăn $\left(Z_{1}\right)$, thuốc $\left(Z_{2}\right)$ và nhiên liệu $\left(Z_{3}\right)$ và 02 biến đầu vào thông thường là giống $\left(X_{1}\right)$ và lao động $\left(X_{2}\right)$. Như vậy 
để cụ thể hóa phương pháp phân tích và tăng tính ứng dụng cho người đọc, bài viết sẽ giới thiệu chi tiết về quá trình ước lượng hiệu quả môi trường trong trường hợp có 5 yếu tố đầu vào, gồm ba yếu tố có ảnh hưởng xấu đến môi trường và hai yếu tố đầu vào thông thường như sau:

Hàm sản xuất biên ngẫu nhiên translog của mô hình nuôi tôm với 02 đầu vào thông thường và 03 đầu vào có ảnh hưởng xấu đến môi trường được viết lại như sau:

$$
\begin{aligned}
\operatorname{Ln} Y_{i}=\beta_{0}+ & \beta_{1} \ln X_{1}+\beta_{2} \ln X_{2}+\alpha_{1} \ln Z_{1}+\alpha_{2} \ln Z_{2}+\alpha_{3} \ln Z_{3} \\
& +1 / 2 \beta_{11} \ln X_{1} \ln X_{1}+1 / 2 \beta_{22} \ln X_{2} \ln X_{2}+1 / 2 \alpha_{11} \ln Z_{1} \ln Z_{1} \\
& +1 / 2 \alpha_{22} \ln Z_{2} \ln Z_{2}+1 / 2 \alpha_{33} \ln Z_{3} \ln Z_{3}+\beta_{12} \ln X_{1} \ln X_{2} \\
& +\delta_{11} \ln X_{1} \ln Z_{1}+\delta_{12} \ln X_{1} \ln Z_{2}+\delta_{13} \ln X_{1} \ln Z_{3}+\delta_{21} \ln X_{2} \ln Z_{1} \\
& +\delta_{22} \ln X_{2} \ln Z_{2}+\delta_{23} \ln X_{2} \ln Z_{3}+\delta_{31} \ln Z_{1} \ln Z_{2}+\delta_{32} \ln Z_{1} \ln Z_{3} \\
& +\delta_{41} \ln Z_{2} \ln Z_{3}+v_{i}-u_{i}
\end{aligned}
$$

Lưu ý: Tổng số biến kết hợp của các biến độc lập được sử dụng trong hàm translog được tính theo công thức sau: $N=\frac{k(k+3)}{2}$, trong đó $\mathrm{k}$ là số biến độc lập và $\mathrm{N}$ là tổng số kết hợp. Như vậy, trong trường hợp này, tổng số kết hợp là $N=\frac{5(5+3)}{2}=20$.

Như vậy hiệu quả môi trường (khả năng giảm thức ăn, thuốc và nhiên liệu) được tính bằng cách thay thế $Z_{1}, Z_{2}$ và $Z_{3}$ lần lượt bằng $\Phi Z_{1}, \Phi Z_{2}$ và $\Phi Z_{3}$ và cho $u_{i}=0$, kết quả ta được

$$
\begin{aligned}
\operatorname{Ln} Y_{i}= & \beta_{0}+\beta_{1} \ln X_{1}+\beta_{2} \ln X_{2}+\alpha_{1} \ln \Phi Z_{1}+\alpha_{2} \ln \Phi Z_{2}+\alpha_{3} \ln \Phi Z_{3} \\
& +1 / 2 \beta_{11} \ln X_{1} \ln X_{1}+1 / 2 \beta_{22} \ln X_{2} \ln X_{2}+1 / 2 \alpha_{11} \ln \Phi Z_{1} \ln \Phi Z_{1} \\
& +1 / 2 \alpha_{22} \ln \Phi Z_{2} \ln \Phi Z_{2}+1 / 2 \alpha_{33} \ln \Phi Z_{3} \ln \Phi Z_{3}+\beta_{12} \ln X_{1} \ln X_{2} \\
& +\delta_{11} \ln X_{1} \ln \Phi Z_{1}+\delta_{12} \ln X_{1} \ln \Phi Z_{2}+\delta_{13} \ln X_{1} \ln \Phi Z_{3} \\
& +\delta_{21} \ln X_{2} \ln \Phi Z_{1}+\delta_{22} \ln X_{2} \ln \Phi Z_{2}+\delta_{23} \ln X_{2} \ln \Phi Z_{3} \\
& +\delta_{31} \ln \Phi Z_{1} \ln \Phi Z_{2}+\delta_{32} \ln \Phi Z_{1} \ln \Phi Z_{3}+\delta_{41} \ln \Phi Z_{2} \ln \Phi Z_{3}+v_{i}
\end{aligned}
$$

Cho (8) và (9) bằng nhau ta có

$$
\begin{aligned}
\left(\alpha_{1} \ln \Phi Z_{1}-\right. & \left.\alpha_{1} \ln Z_{1}\right)+\left(\alpha_{2} \ln \Phi Z_{2}-\alpha_{2} \ln Z_{2}\right)+\left(\alpha_{3} \ln \Phi Z_{3}-\alpha_{3} \ln Z_{3}\right) \\
& +\left(1 / 2 \alpha_{11} \ln \Phi Z_{1} \ln \Phi Z_{1}-1 / 2 \alpha_{11} \ln Z_{1} \ln Z_{1}\right) \\
& +\left(1 / 2 \alpha_{22} \ln \Phi Z_{2} \ln \Phi Z_{2}-1 / 2 \alpha_{22} \ln Z_{2} \ln Z_{2}\right) \\
& +\left(1 / 2 \alpha_{33} \ln \Phi Z_{3} \ln \Phi Z_{3}-1 / 2 \alpha_{33} \ln Z_{3} \ln Z_{3}\right) \\
& +\left(\delta_{11} \ln X_{1} \ln \Phi Z_{1}-\delta_{11} \ln X_{1} \ln Z_{1}\right) \\
& +\left(\delta_{12} \ln X_{1} \ln \Phi Z_{2}-\delta_{12} \ln X_{1} \ln Z_{2}\right) \\
& +\left(\delta_{13} \ln X_{1} \ln \Phi Z_{3}-\delta_{13} \ln X_{1} \ln Z_{3}\right) \\
& +\left(\delta_{21} \ln X_{2} \ln \Phi Z_{1}-\delta_{21} \ln X_{2} \ln Z_{1}\right) \\
& +\left(\delta_{22} \ln X_{2} \ln \Phi Z_{2}-\delta_{22} \ln X_{2} \ln Z_{2}\right) \\
& +\left(\delta_{23} \ln X_{2} \ln \Phi Z_{3}-\delta_{23} \ln X_{2} \ln Z_{3}\right) \\
& +\left(\delta_{31} \ln \Phi Z_{1} \ln \Phi Z_{2}-\delta_{31} \ln Z_{1} \ln Z_{2}\right) \\
& +\left(\delta_{32} \ln \Phi Z_{1} \ln \Phi Z_{3}-\delta_{32} \ln Z_{1} \ln Z_{3}\right) \\
& +\left(\delta_{41} \ln \Phi Z_{2} \ln \Phi Z_{3}-\delta_{41} \ln Z_{2} \ln Z_{3}\right)+u_{i}=0
\end{aligned}
$$

Bằng một số phép tính toán logarit và biến đổi ta được 


$$
\begin{aligned}
\alpha_{1} \ln E E_{i}+\alpha_{2} & \ln E E_{i}+\alpha_{3} \ln E E_{i} \\
& +\left[1 / 2 \alpha_{11}\left(\ln E E_{i}\right)^{2}+1 / 2 \alpha_{11} \ln E E_{i}\left(\ln Z_{1}+\ln Z_{1}\right)\right] \\
& +\left[1 / 2 \alpha_{22}\left(\ln E E_{i}\right)^{2}+1 / 2 \alpha_{22} \ln E E_{i}\left(\ln Z_{2}+\ln Z_{2}\right)\right] \\
& +\left[1 / 2 \alpha_{33}\left(\ln E E_{i}\right)^{2}+1 / 2 \alpha_{33} \ln E E_{i}\left(\ln Z_{3}+\ln Z_{3}\right)\right] \\
& +\delta_{11} \ln X_{1} \ln E E_{i}+\delta_{12} \ln X_{1} \ln E E_{i}+\delta_{13} \ln X_{1} \ln E E_{i} \\
& +\delta_{21} \ln X_{2} \ln E E_{i}+\delta_{22} \ln X_{2} \ln E E_{i}+\delta_{23} \ln X_{2} \ln E E_{i} \\
& +\left[\delta_{31}\left(\ln E E_{i}\right)^{2}+\delta_{31} \ln E E_{i}\left(\ln Z_{1}+\ln Z_{2}\right)\right] \\
& +\left[\delta_{32}\left(\ln E E_{i}\right)^{2}+\delta_{32} \ln E E_{i}\left(\ln Z_{1}+\ln Z_{3}\right)\right] \\
& +\left[\delta_{41}\left(\ln E E_{i}\right)^{2}+\delta_{41} \ln E E_{i}\left(\ln Z_{2}+\ln Z_{3}\right)\right]+u_{i}=0
\end{aligned}
$$

Từ phương trình (11), ta được

$$
\begin{array}{r}
\left(1 / 2 \alpha_{11}+1 / 2 \alpha_{22}+1 / 2 \alpha_{33}+\delta_{31}+\delta_{32}+\delta_{41}\right)\left(\ln E E_{i}\right)^{2}+\left[\alpha_{1}+\right. \\
\alpha_{2}+1 / 2 \alpha_{11}\left(\ln Z_{1}+\ln Z_{1}\right)+1 / 2 \alpha_{22}\left(\ln Z_{2}+\ln Z_{2}\right)+1 / 2 \alpha_{33}\left(\ln Z_{3}+\right. \\
\left.\ln Z_{3}\right)+\delta_{11} \ln X_{1}+\delta_{12} \ln X_{1}+\delta_{13} \ln X_{1}+\delta_{21} \ln X_{2}+\delta_{22} \ln X_{2}+\delta_{23} \ln X_{2}+ \\
\left.\delta_{31}\left(\ln Z_{1}+\ln Z_{2}\right)+\delta_{32}\left(\ln Z_{1}+\ln Z_{3}\right)+\delta_{41}\left(\ln Z_{2}+\ln Z_{3}\right)\right] \ln E E_{i}+u_{i}=0
\end{array}
$$

Như vậy hiệu quả môi trường được tính bằng công thức sau:

$$
E E_{i}=\exp \left(\frac{-b_{i}+\sqrt{b_{i}^{2}-4 a_{i} u_{i}}}{2 a_{i}}\right)
$$

Với $a_{i}=1 / 2 \alpha_{11}+1 / 2 \alpha_{22}+1 / 2 \alpha_{33}+\delta_{31}+\delta_{32}+\delta_{41} \quad \forall a_{i} \neq 0$;

$$
\begin{aligned}
b_{i}=\alpha_{1}+\alpha_{2}+ & 1 / 2 \alpha_{11}\left(\ln Z_{1}+\ln Z_{1}\right)+1 / 2 \alpha_{22}\left(\ln Z_{2}+\ln Z_{2}\right)+1 / 2 \alpha_{33}\left(\ln Z_{3}+\ln Z_{3}\right) \\
& +\delta_{11} \ln X_{1}+\delta_{12} \ln X_{1}+\delta_{13} \ln X_{1}+\delta_{21} \ln X_{2}+\delta_{22} \ln X_{2}+\delta_{23} \ln X_{2} \\
& +\delta_{31}\left(\ln Z_{1}+\ln Z_{2}\right)+\delta_{32}\left(\ln Z_{1}+\ln Z_{3}\right)+\delta_{41}\left(\ln Z_{2}+\ln Z_{3}\right)
\end{aligned}
$$

\section{2. Ước lựng hiệu quả môi truờng}

Để ước lượng được hiệu quả môi trường hay khả năng giảm các yếu tố đầu vào có ảnh hưởng xấu đến môi trường, trước hết cần ước lượng hàm sản xuất biên để mô tả công nghệ sản xuất của nông hộ nuôi tôm. Thống kê mô tả về đầu vào và đầu ra của mô hình nuôi tôm được sử dụng để ước lượng hàm sản xuất translog được trình bày ở Bảng 1 sau:

\section{Bảng 1}

Đầu vào và đầu ra của mô hình tôm chuyển đổi

\begin{tabular}{cccrrrr}
\hline Tiêu chí & Ký hiệu & \multicolumn{1}{c}{ Đơn vị } & Trung bình & Lệch chuẩn & Nhỏ nhất & Lớn nhất \\
\hline Năng suất $^{1}$ & $Y$ & $\mathrm{Kg} / \mathrm{ha} / \mathrm{vụ}$ & $3.807,96$ & $4.545,23$ & 75 & $22.727,27$
\end{tabular}

\footnotetext{
${ }^{1}$ Biến đầu ra của quá trình sản xuất trong nghiên cứu này là năng suất tôm trung bình cho 01 ha/vụ/năm. Các đầu ra khác trong quá trình sản xuất sẽ không được xem xét trong nghiên cứu này do mức độ rất ít và mục tiêu chính của nông hộ là tôm nên các đầu ra khác như cá, cua có thể gọi là kết quả của sự may mắn do thiên nhiên ưu đãi, không phản ánh được công nghệ sản xuất của nông hộ.
} 


\begin{tabular}{llcrrrr} 
Thức ăn & & & & \\
Thuốc & $Z_{1}$ & $\mathrm{Kg} / \mathrm{ha} / \mathrm{vu}$ & $3.3442,10$ & $29.169,10$ & $2.857,14$ & 100.000 \\
Nhiên liệu & $Z_{2}$ & Ngàn/ha/vụ & $15.738,22$ & $26.776,53$ & 113,39 & $147.058,8$ \\
Giống & $Z_{3}$ & Ngàn/ha/vụ & $19.548,33$ & $24.246,55$ & $1.214,29$ & 130.400 \\
Lao động & $X_{1}$ & Con/ha/vụ & $449.864,20$ & $580.522,20$ & 100.000 & 3.000 .000 \\
\hline
\end{tabular}

Nguồn: Kết quả điều tra năm 2017, n=67

Kết quả Bảng 1 cho thấy các chỉ tiêu về đầu vào và đầu ra trong địa bàn nghiên cứu có mức độ biến thiên khá lớn. Sự biến động lớn này là do ảnh hưởng của điều kiện sản xuất, mức độ thâm canh khác nhau, trình độ quản lý và nguồn con giống của các nông hộ khác nhau nên mức độ thành công cũng như thất bại là khác nhau. Sự biến thiên lớn này đã làm cho các tham số được ước lượng trong mô hình SFA bằng phương pháp ước lượng hợp lý tối đa (MLE) ít có ý nghĩa. Dựa trên kết quả kiểm định log-likelihood (LR test), hàm sản xuất biên ngẫu nhiên translog được chấp nhận. Kết quả ước lượng hàm sản xuất biên ngẫu nhiên translog được trình bày trong Bảng 2 sau.

\section{Bảng 2}

Ước lượng hàm sản xuất biên ngẫu nhiên translog bằng MLE

\begin{tabular}{|c|c|c|c|c|c|}
\hline & \multicolumn{2}{|c|}{ MLE } & \multicolumn{3}{|c|}{ MLE } \\
\hline Biến độc lập & Hệ số gốc & $\begin{array}{l}\text { Sai số } \\
\text { chuẩn }\end{array}$ & Biến độc lập & Hệ số gốc & $\begin{array}{l}\text { Sai số } \\
\text { chuẩn }\end{array}$ \\
\hline $\ln Z_{1}$ & 0,265 & 2,391 & $\left(\ln Z_{2} \ln Z_{2}\right) / 2$ & 0.055 & 0.065 \\
\hline $\ln Z_{2}$ & $1,420^{*}$ & 0,886 & $\ln Z_{2} \ln Z_{3}$ & 0.034 & 0.064 \\
\hline $\ln Z_{3}$ & $-0,021$ & 1,651 & $\ln Z_{2} \ln X_{1}$ & -0.130 & 0.099 \\
\hline $\ln X_{1}$ & $-9,973^{* * *}$ & 2,930 & $\ln Z_{2} \ln X_{2}$ & 0.028 & 0.066 \\
\hline $\ln X_{2}$ & $3,523^{* * *}$ & 1,164 & $\left(\ln Z_{3} \ln Z_{3}\right) / 2$ & 0.088 & 0.157 \\
\hline$\left(\ln Z_{1} \ln Z_{1}\right) / 2$ & $-0,147$ & 0,280 & $\ln Z_{3} \ln X_{1}$ & -0.196 & 0.164 \\
\hline $\ln Z_{1} \ln Z_{2}$ & $-0,052$ & 0,077 & $\ln Z_{3} \ln X_{2}$ & 0.093 & 0.096 \\
\hline $\ln Z_{1} \ln Z_{3}$ & 0,093 & 0,082 & $\left(\ln X_{1} \ln X_{1}\right) / 2$ & $1.011^{* * *}$ & 0.286 \\
\hline $\ln Z_{1} \ln X_{1}$ & 0,128 & 0,167 & $\ln \mathrm{X}_{1} \ln \mathrm{X}_{2}$ & $-0.263^{*}$ & 0.128 \\
\hline $\ln Z_{1} \ln X_{2}$ & $-0,040$ & 0,116 & $\left(\ln \mathrm{X}_{2} \ln \mathrm{X}_{2}\right) / 2$ & -0.137 & 0.177 \\
\hline$\lambda$ & 1,357 & 0,259 & Wald $\chi^{2}$ value & 229.16 & \\
\hline Log Likelihood & $-55,569$ & & & & \\
\hline
\end{tabular}

Nguồn: Số liệu điều tra năm 2017, n=67

Từ kết quả các tham số ước lượng ở Bảng 2 và áp dụng công thức (7) trong trường hợp tổng quát hay công thức (13) trong trường hợp mô hình có 5 yếu tố đầu vào, trong đó có 3 yếu tố được xem là có ảnh hưởng xấu đến môi trường (thức ăn, thuốc và nhiên liệu) ta có thể tính được hiệu quả môi trường của các nông hộ nuôi tôm. Để người đọc có thể dễ theo dõi và sử dụng các công thức trên, kết quả ước lượng hiệu quả kỹ thuật và hiệu quả môi trường của 10 nông hộ đầu tiên trong dãy số liệu được trình bày chung với kết quả tổng hợp về ước lượng hiệu quả môi trường cho nông hộ nuôi tôm thâm canh vùng chuyển đổi tỉnh Kiên Giang, kết

\footnotetext{
${ }^{2}$ Theo Le và Ong (2010) thì các nhà máy sản xuất thức ăn đều theo tiêu chuẩn của Bộ đưa ra theo từng giai đoạn phát triển của tôm, mỗi nhà máy thức ăn có 6 - 8 loại thức ăn, trong đó độ đạm dao động từ 36 - 42\% sự dao động này không đáng kể nên biến số thức ăn được xem như khác biệt không có ý nghĩa giữa các loại.
} 
quả lần lượt được trình bày ở Bảng 3 và 4 sau:

\section{Bảng 3}

Kết quả ước lượng hiệu quả kỹ thuật và môi trường của mỗi nông hộ

\begin{tabular}{cccccc}
\hline $\mathbf{H} \hat{\mathbf{o}}$ & $\mathbf{u}_{\mathbf{i}}$ & $\mathbf{T E}$ & Hạng & $\mathbf{E E}_{\mathbf{i}}$ & Hạng \\
\hline 1 & 0,302604 & 75,60 & 2 & 53,44 & 3 \\
2 & 0,683856 & 53,10 & 9 & 23,84 & 8 \\
3 & 0,577700 & 58,72 & 7 & 52,94 & 4 \\
4 & 1,814863 & 17,30 & 10 & 10,81 & 10 \\
5 & 0,571822 & 59,04 & 6 & 20,68 & 9 \\
6 & 0,413587 & 68,36 & 4 & 49,47 & 6 \\
7 & 0,225242 & 81,07 & 1 & 72,35 & 2 \\
8 & 0,419349 & 68,00 & 5 & 52,87 & 5 \\
9 & 0,620909 & 56,38 & 8 & 32,70 & 7 \\
10 & 0,338571 & 73,19 & 3 & 77,64 & 1 \\
\hline Trung bình & & 61,07 & & 42,12 & \\
\hline
\end{tabular}

Nguồn: Số liệu điều tra năm 2017, n=67

Lưu ý: $u_{i}$ là sai số thể hiện sự không hiệu quả về kỹ thuật định hướng đầu ra, là kết quả của mô hình sản xuất biên ngẫu nhiên; $\mathrm{TE}_{\mathrm{i}}=\exp \left(-u_{i}\right)$ là hiệu quả kỹ thuật định hướng đầu ra, được tính theo công thức (2); $\mathrm{EE}_{\mathrm{i}}$ là hiệu quả môi trường của từng hộ được tính dựa vào công thức (7) hoặc (13) và kết quả của Bảng 1 và Bảng 2 .

Từ kết quả Bảng 3 cho thấy hiệu quả môi trường sẽ luôn nhỏ hơn hiệu quả kỹ thuật định hướng đầu ra và hai loại hiệu quả phân bố độc lập nhau. Hiệu kỹ thuật định hướng đầu ra trung bình của 10 hộ là $61,07 \%$, có nghĩa là ở mức đầu vào hiện tại nông hộ có thể tăng thêm được khoảng 39\% đầu ra. Hiệu quả môi trường trung bình của 10 hộ là $42,12 \%$, kết quả này cho thấy nông hộ có thể giảm được khoảng 58\% tổng đầu vào là thức ăn, thuốc và nhiên liệu mà vẫn giữ được mức đầu ra hiện tại.

Kết quả ước lượng Bảng 4 cho thấy hiệu quả môi trường trung bình của nông hộ nuôi tôm vùng chuyển đổi tỉnh Kiên Giang là $52,79 \%$, kết quả này cho thấy nông hộ có thể giảm đến $47,21 \%$ lượng đầu vào có ảnh hưởng xấu đến môi trường gồm thức ăn, thuốc và nhiên liệu. Kết quả nghiên cứu cũng cho thấy có sự khác biệt lớn về hiệu quả môi trường giữa các nông hộ trong địa bàn nghiên cứu. Sự khác biệt lớn này một phần do mô hình mới chuyển đổi nên nông hộ sử dụng chưa hiệu quả các yếu tố đầu vào và mức độ thâm canh khác nhau giữa các hộ nên sự đầu tư cũng còn khác biệt lớn. Thêm vào đó, do là mô hình mới nên mức độ thành công cũng như rủi ro giữa các hộ cũng có sự khác biệt lớn.

\section{Bảng 4}

Kết quả tổng hợp ước lượng hiệu quả môi trường của nông hộ nuôi tôm

\begin{tabular}{cccc}
\hline Hiệu quả môi trường (\%) & Tần suất & Phần trăm (\%) & \% tích lũy \\
\hline 90 & 0 & 0,00 & 0 \\
$80-90$ & 3 & 4,48 & 4,48 \\
$70-80$ & 10 & 14,93 & 19,41 \\
$60-70$ & 8 & 11,94 & 31,35
\end{tabular}




\begin{tabular}{ccrc}
$50-60$ & 21 & 31,34 & 62,69 \\
$40-50$ & 10 & 14,93 & 77,62 \\
$30-40$ & 4 & 5,97 & 83,59 \\
$20-30$ & 4 & 5,97 & 89,56 \\
$10-20$ & 7 & 10,44 & 100 \\
$<10$ & 0 & 0,00 & 100 \\
\hline Trung bình & & 52,79 & \\
Nhỏ nhất & 10,50 & \\
Lớn nhất & & 88,23 & \\
\hline
\end{tabular}

Nguồn: Kết quả phân tích dữ liệu của nhóm nghiên cứu

\section{Kết luận}

Đo lường hiệu quả môi trường bằng cách sử dụng phương pháp tiếp cận SFA là một việc làm cần thiết trong thời gian tới do nhu cầu về sản phẩm sạch, an toàn và tính bền vững về môi trường ngày càng tăng. Hiệu quả môi trường sẽ góp phần phản ánh mức độ giảm thiểu các yếu tố đầu vào có ảnh hưởng xấu đến môi trường trong điều kiện đầu vào khác và đầu ra không thay đổi. Kết quả nghiên cứu cho thấy hiệu quả môi trường trung bình của mô hình nuôi tôm thâm canh vùng chuyển đổi tỉnh Kiên Giang là 52,79 , cho thấy nông hộ vẫn chưa sử dụng hiệu quả các yếu tố đầu vào có ảnh hưởng xấu đến môi trường hay nói cách khác nông hộ có thể giảm khoảng $47,21 \%$ tổng lượng đầu vào các yếu tố thức ăn, thuốc và nhiên liệu. Kết quả nghiên cứu cũng cho thấy có sự khác biệt lớn về hiệu quả môi trường giữa các nông hộ nuôi tôm trong địa bàn.

\section{Tài liệu tham khảo}

Billi, A., Canitano, G., \& Quarto, A. (2007). The economics of water efficiency: A review of theories, measurement issues and integrated models. Retrieved June 20, 2017, from https://om.ciheam.org/article.php?IDPDF=800791

Carew-Reid, J. (2008). Rapid assessment of the extent and impact of sea level rise in Viet Nam. Queensland, Australia: International Centre for Environment Management (ICEM).

Caves, D. W., Christensen, L. R., \& Diewert, W. E. (1982). Multilateral comparisons of output, input, and productivity using superlative index numbers. The Economic Journal, 92(365), 73-86.

Dang, N. K., Nguyen, B. V., \& Nguyen, T. H. (2007). Water use and competition in the Mekong Delta, Vietnam. In Challenges to sustainable development in the Mekong Delta: Regional and national policy issues and research needs (pp. 143-188).

Färe, R., Grosskopf, S., Lovell, C. K., \& Pasurka, C. (1989). Multilateral productivity comparisons when some outputs are undesirable: A nonparametric approach. The Review of Economics and Statistics, 71(1), 90-98.

GSO. (2013). Statistical yearbook of Vietnam. Retrieved June 27, 2017, from General Statistics Office website: https://www.gso.gov.vn/en/data-and-statistics/2019/10/statiscalyearbook-of-vietnam-2013/ 
Heong, K. L., \& Hardy, B. (2009). Planthoppers: New threats to the sustainability of intensive rice production systems in Asia. Los Baños, Philippines: International Rice Research Institute.

Le, H. T., \& Ong, Q. M. (2010). Hiện trạng sủ dụng và quản lý thức ăn nuôi tôm thẻ chân trắng (litopenaeus vannamei) ơ Việt Nam [Present status of feed use and feed mangement in white leg shrimp (litopenaeus vannamei) farming in Vietnam]. Retrieved May 20, 2018, from http://fof.hcmuaf.edu.vn/data/file/1_\%20LT\%20Hung\%20_\%20OM\%20QuyDHNL-Hien\%20trang\%20su\%20dung\%20thuc\%20an__tom\%20chan\%20trang.pdf

Ministry of Agriculture and Rural Development (MARD). (2014). Quyết định số 3824/QĐBNN-TCTS ban hành quy phạm thực hành nuôi trồng thủy sản tốt Việt Nam (VIETGAP) [Decision No. 3824 / QD-BNN-TCTS promulgating good aquaculture practices in Vietnam]. Retrieved May 10, 2018, from https://thuvienphapluat.vn/van-ban/Tainguyen-Moi-truong/Quyet-dinh-5345-QD-BNN-TCLN-2014-huong-dan-thuc-hien1938-QD-TTg-nang-cao-nang-luc-phong-chay-chua-chay-rung-261553.aspx

Nguyen, B. T., Lam, H., \& Thach, P. S. (2012). Đánh giá tính tổn thương do xâm nhập mặn ở đồng bằng sông Cửu Long [Vulnerability assessment due to saline intrusion in the Mekong Delta]. Tạp chí Khoa học Trường Đại học Cần Tho, 24b, 229-239.

Nguyen, C. D. (2011). Transformation of farming systems in coastal Mekong delta: Seeking for a better management and sustainability. Viet Nam Socio-Economic Development, 65.

Nguyen, D. H., \& Tran, D. T. T. (1999). Economic and health consequences of pesticide use in paddy production in the Mekong Delta, Vietnam. Retrieved June 25, 2017, from https://www.car.chula.ac.th/display7.php?bib=b1515853

Nguyen, H. B., \& Yabe, M. (2017). Improvement in irrigation water use efficiency: A strategy for climate change adaptation and sustainable development of Vietnamese tea production. Environment, Development and Sustainability, 19(4), 1247-1263.

Nguyen, H. B., Takahashi, Y., \& Yabe, M. (2016). Environmental efficiency and economic losses of Vietnamese tea production: Implications for cost savings and environmental protection. Journal- Faculty of Agriculture Kyushu University, 61(2), 383-390.

Pittman, R. W. (1983). Multilateral productivity comparisons with undesirable outputs. The Economic Journal, 93(372), 883-891.

Reinhard, S., \& Thijssen, G. (2000). Nitrogen efficiency of Dutch dairy farms: A shadow cost system approach. European Review of Agricultural Economics, 27(2), 167-186.

Reinhard, S., Lovell, C. K., \& Thijssen, G. (1999). Econometric estimation of technical and environmental efficiency: An application to Dutch dairy farms. American Journal of Agricultural Economics, 81(1), 44-60.

Reinhard, S., Lovell, C. K., \& Thijssen, G. (2000). Environmental efficiency with multiple environmentally detrimental variables; estimated with SFA and DEA. European Journal of Operational Research, 121(2), 287-303.

Sharma, B., Molden, D., \& Cook, S. (2015). Water use efficiency in agriculture: Measurement, current situation and trends. In P. Drechsel, P. Heffer, H. Magan, R. Mikkelsen \& D. Wichlens (Eds.), Managing water and fertilizer for sustainable agricultural intensification (pp. 39-64). 
Vo, T. H. (2015). Ứng dụng phương pháp phân tích giới hạn sản xuất ngẫu nhiên để đo lường hiệu quả môi trường của hoạt động sản xuất nông nghiệp [Apply random production limit analysis method to measure the environmental efficiency of agricultural production activities]. Tạp chí Khoa học và Phát triển, 13(8), 1519-1526.

Vo, T. H. (2017). Resource use efficiency and economic losses: Implications for sustainable rice production in Vietnam. Environment, Development and Sustainability, 19(1), 1-16. doi:10.1007/s10668-015-9724-0

Vo, T. H., \& Yabe, M. (2015). Technical efficiency of ecologically engineered rice production in the Mekong Delta of Vietnam: Application of SFA. Global Journal of Science Frontier Research, 15(5).

Vo, T. H., Nguyen, C. D., Takahashi, Y., \& Yabe, M. (2018). Water use efficiency in rice production: Implications for climate change adaptation in the Vietnamese Mekong Delta. Process Integration and Optimization for Sustainability, 2(3), 1-18.

Vo, T. H., Nguyen, C. D., Takahashi, Y., Kopp, S. W., \& Yabe, M. (2018). Technical and environmental efficiency of eco-friendly rice production in the upstream region of the Vietnamese Mekong Delta. Environment, Development and Sustainability, 21(1), 1-24.

Vo, T. H., Yabe, M., Nguyen, T. T., \& Huynh, K. V. (2015). Environmental efficiency of ecologically engineered rice production in the Mekong Delta of Vietnam. JournalFaculty of Agriculture Kyushu University, 60(2), 493-500.

Wassmann, R., Nguyen, H. X., Chu, H. T., \& To, T. P. (2004). Sea level rise affecting the Vietnamese Mekong Delta: Water elevation in the flood season and implications for rice production. Climatic Change, 66(1/2), 89-107. 